\title{
Generation of an Attenuated, Cross-Protective Pepino mosaic virus Variant Through Alignment-Guided Mutagenesis of the Viral Capsid Protein
}

\author{
Godwill M. Chewachong, Sally A. Miller, Joshua J. Blakeslee, David M. Francis, T. Jack Morris, and Feng Qu
}

First, second, and sixth authors: Department of Plant Pathology, and third and fourth authors: Department of Horticulture and Crop Science, Ohio Agricultural Research and Development Center, The Ohio State University, Wooster 44691; and fifth author: School of Biological Science, University of Nebraska, Lincoln 68583-0900.

Accepted for publication 1 July 2014.

\begin{abstract}
Chewachong, G. M., Miller, S. A., Blakeslee, J. J., Francis, D. M., Morris, T. J., and Qu, F. 2015. Generation of an attenuated, cross-protective Pepino mosaic virus variant through alignment-guided mutagenesis of the viral capsid protein. Phytopathology 105:126-134.

Mild variants of many viruses are able to protect infected plants from subsequent invasion by more severe variants of the same viruses through a process known as cross-protection. In the past, the cross-protective viral variants were commonly derived from mild field isolates that were sometimes genetically heterogeneous, providing variable levels of crossprotection. Here, we report a novel approach to rapidly generate crossprotective variants of the tomato-infecting Pepino mosaic virus (PepMV) independently of the availability of mild field isolates. Our approach sought to attenuate PepMV by mutating less conserved amino acid resi-

dues of the abundantly produced capsid protein (CP). These less-conserved amino acid residues were identified through multiple alignments of CPs of six potexviruses including PepMV, and were altered systematically to yield six PepMV mutants. These mutants were subsequently inoculated onto the model plant Nicotiana benthamiana, as well as tomato, to evaluate their accumulation levels, symptom severities, and cross-protection potentials. The mutant $\mathrm{KD}$, in which the threonine $(\mathrm{T})$ and alanine (A) residues at $\mathrm{CP}$ positions 66 and 67 were replaced with lysine (K) and aspartic acid (D), respectively, were found to accumulate to low levels in infected plants, cause very mild symptoms, and effectively protect both $N$. benthamiana and tomato against secondary infections by wild-type PepMV. These data suggest that our approach represents a simple, fast, and reliable way of generating attenuated viral variants capable of cross-protection.
\end{abstract}

Cross-protection refers to the practice of protecting crop plants against virus invasions by preinoculating the seedlings with a mild isolate of the same virus (14). It was first reported by McKinney, who observed that tobacco plants preinfected with a mild variant of Tobacco mosaic virus (TMV) were protected from infections by a severe TMV strain $(21,37)$. Similar observations have since been made with many other viruses, leading to the adoption of cross-protection to control viruses such as Citrus tristeza virus, Papaya rinspot virus, Zucchini yellow mosaic virus (ZYMV), and Potato virus X (PVX) $(4,5,24,32,34)$. The crossprotection-inducing variant of a given virus typically is asymptomatic or only mildly symptomatic in host plants, yet able to elicit protection against a severe isolate of the same virus $(33,37)$.

Current approaches for selecting cross-protective virus isolates are time consuming because they rely primarily on the observation of mild diseases in the field. Although the mechanism of cross-protection remains poorly understood, it is well established that the mild strain used to elicit cross-protection must have a high level of genetic similarity with the more symptomatic virus strains in order to exert effective protection (27). Hence, it was postulated that engineered attenuation of a severe wild-type (WT) virus could be an ideal approach to obtain the cross-protective viral variants, because the engineered viral variant would represent a homogenous source of inoculum with a high level of sequence identity to its parental virus (15). However, this ap-

Corresponding author: F. Qu; E-mail address: qu.28@osu.edu

http://dx.doi.org/10.1094/PHYTO-01-14-0018-R

(c) 2015 The American Phytopathological Society proach has not yet been thoroughly investigated in plant systems for many viruses. Although engineered attenuation of ZYMV has been reported by two independent groups, both of these studies relied on the sequence information derived from naturally occurring mild isolates of ZYMV to guide their mutagenesis $(8,9,15)$.

Here, we report the development of a simple procedure that enabled us to engineer a mild, cross-protective variant of Pepino mosaic virus (PepMV) without having to rely on previous knowledge of mild isolates of this virus. PepMV is an emerging tomato pathogen and a major yield-limiting factor in tomato production worldwide, causing significant yield losses of greenhouse tomato $(10,11,16,30)$. PepMV belongs to the genus Potexvirus, family Flexiviridae, with nonenveloped, flexuous rodshaped virions of $\approx 580 \mathrm{~nm}$ in length $(1,13)$ (Fig. 1A). Its singlestranded, positive-sense (+) RNA genome is $\approx 6.4 \mathrm{~kb}$ long, encoding five open reading frames (ORFs) (Fig. 1B). The ORF1 encodes the 164-kDa viral RNA-dependent RNA polymerase (RdRp), whereas the partially overlapping ORFs 2 to 4 encode the triple gene block (TGB) proteins involved in viral cell-to-cell movement. Finally, ORF5 encodes the $25-\mathrm{kDa}$ capsid protein (CP). The gene expression strategy of PepMV is thought to be similar to that of other potexviruses, with the viral RdRp translated directly from the genomic RNA (gRNA), whereas TGB proteins and CP are from three subgenomic RNAs (31).

In this study, we sought to test the feasibility of engineering attenuated, cross-protective PepMV variants through targeted mutagenesis based on alignments of CPs of multiple potexviruses. One mutant, designated KD, was considerably attenuated, yet infected both Nicotiana benthamiana and tomato plants systemically. Strikingly, it protected the infected plants against secondary 
infection by WT PepMV. In addition, KD remained stable both in terms of the mildness of symptoms induced and the identity of the mutated nucleotides for at least five passages. This approach of plant virus attenuation, designated alignment-guided mutagenesis, does not require prior knowledge of any naturally occurring mild isolates. Therefore, it is expected to be applicable to other plant viruses, leading to more widespread adoption of cross-protection for virus disease control and improved productivity in crops prone to virus diseases.

\section{MATERIALS AND METHODS}

Plant materials, growth conditions, and virus source. $N$. benthamiana and tomato plants (14 cultivars) were grown in a growth chamber set at $22^{\circ} \mathrm{C}$, with $14 \mathrm{~h}$ of daylight. Adult tomato plants were sometimes reared in greenhouse rooms under ambient conditions. The PepMV isolate used in this study was obtained from a symptomatic tomato plant sent to the Morris lab at the University of Nebraska-Lincoln in 2006.

Synthesis and cloning of the full-length cDNA of the PepMV Nebraska isolate. Virus-specific double-stranded RNA (dsRNA) was then purified from $N$. benthamiana infected with the PepMV Nebraska isolate (PepMV-NE) and used to generate short cDNA fragments by reverse-transcription-polymerase chain reaction (RT-PCR) using random hexamers as primers (20). Sequence analysis of these short cDNA fragments identified this virus as PepMV. The full-length sequence of PepMV-NE was obtained by sequencing overlapping RT-PCR fragments generated with specific primers (Table 1) and deposited to GenBank (accession number: KF281605). The full-length cDNA was then RT-PCR amplified as two overlapping fragments, assembled, and cloned downstream of a T7 promoter in a plasmid vector derived from pBlueScript SK. A stretch of 30 "T"'s was included in the 3' end reverse primer to enable the addition of the poly-A tail to the
A

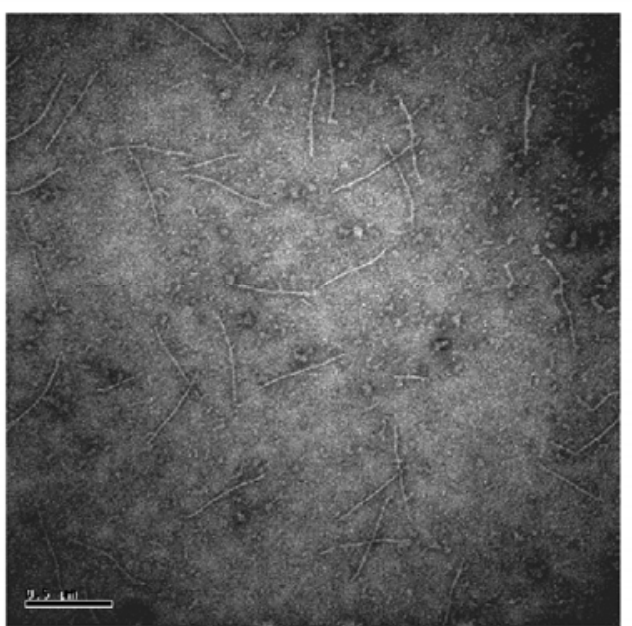

\section{C}

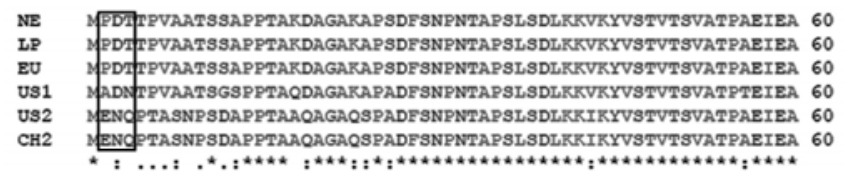

NE LGKIF TA GLAANETGPAMDLARAYADVOSSKSAOLIGATPSNPALSRRALAAOFDF TN 120 IP LGKI TA IGLANAETGPAMWDLARAYADVOSSKSAOLIGATPSNPALSRRALAAOFDF IN 120 EU IGKI TA IGLANETEPAMEDLARAYADVQSSKSAOLIGATPSNPALSRRALAAQFDA IN 120 US1 IGKI FA IGLAANETGPANDLARAYADVQSSKSAOLIGATPSNPALSRRALAAOFDE IN 120 US2 IGKI FA LGLAANETGPAMWDLARAYADVOSSKSAOLIGATPSNPALSRRALAAOFDF IN 120 CH2 LGKI ERA IGLAANETGPAMWDLARAYADVQSSKSAQLIGATPSNPALSRRALAAQFDE EN 120

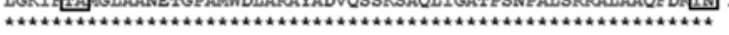

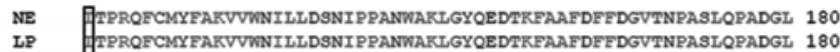
IP TPRQFCMYFAKVVWNILLDSNIPPANWARLGYOEDTKFAAFDFFDGVTNPASLQPADGL 180 EU TPRQFCMYFAKV VWNILLDSNI P PANWAKL GYOEDNKFAAFDFFDGVTNPASLQPADGL 180 U31 PPRQFCMYFAKVVWNILIDSNIPPANWAKLGYOEDTKFAAFDFFDGVTNPASLQPADGL 180

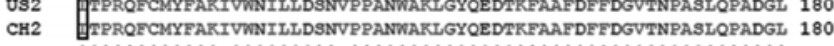

NE IRQPNEKELAAHSVAKYGALARORISTGNYITTLGEVTRGHMGGANTMYAIDAPIEI 237 LP IRQPNEKELAAHSVARYGALARQRISTGNYITTLGEVTRGHMGGANTMYAIDAPEEL 237 EU IROPNEKELAAHSVAKYGALARORISTGNYITTLGEVTRGHMGGANTMYAIDAPEEL 237 US1 IRQPNEKELAAHSVAKYGALARORISTGNYITTLGEVTRGHMGGANTMYAIDAPIEI 237 US2 IROPNEKELAAHSVARYGALARORISTGNYITTLGEVTRGHMGGANTYYAIDAPTEL 237 CH2 IRQPEKELAHSVARYGALARQRISTGNYITTLGEVTRGHNGGANTMYAIDAPEEI 237 IROPNEKELAAHSVAKYGALARORISTGNYITTLGEVTRGHMGGANTMYAIDAPEEL 237
B

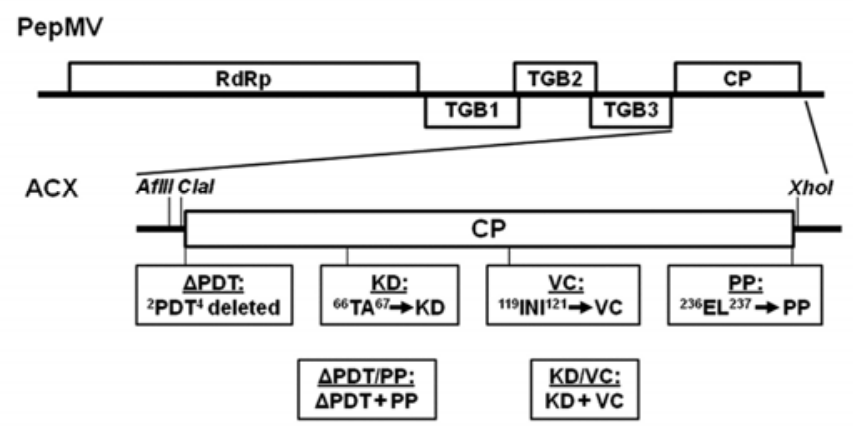

D

Fig. 1. Selection of variable amino acid residues in Pepino mosaic virus (PepMV) capsid protein (CP) as targets for mutagenesis. A, Electron micrograph (EM) of PepMV-NE virions. Homogenates of virus-containing Nicotiana benthamiana leaf sections were applied to EM grids and negatively stained prior to EM observations. B, Genome organization of PepMV, with the approximate positions of five open reading frames shown. Immediately underneath is the expanded CPcoding region the ACX mutant, which contains the new AflII, ClaI, and XhoI sites to accommodate the cloning of mutated CP cDNA. Six CP mutants, with their names and the corresponding amino acid changes detailed in rectangular boxes, are illustrated at the bottom. C, Multiple alignments of amino acid sequences of CPs of six PepMV isolates, with boxes highlighting amino acids identified in the subsequent alignments (see D); asterisks $(*)$ denote invariable amino acids, whereas "." and "." denote amino acids with high or low levels of structural similarities, respectively. D, Multiple alignments of amino acid sequences of six potexviral CPs, with boxes highlighting the variable amino acids that were subjected to mutagenesis in the current study. 
$3^{\prime}$ end of transcribed RNA (Table 1). The resulting PepMV infectious clone was named pPEP8.

Multiple alignment of the $\mathrm{CP}$ sequences of six potexviruses. The amino acid sequence of the PepMV CP was compared with that of five closely related potexviruses using the CLUSTAL 2.1 algorithm (http://www.clustal.org/clustal2/). First, PepMV CP was used as the query to search for closely related potexviruses in GenBank, leading to the identification of five viruses whose CPs share high levels of similarity with PepMV: Narcissus mosaic virus (NarMV; AAP51016), Alstroemeria virus X (AlsVX; YP_319831.1), Lettuce virus X (LetVX; YP_00190944.1), Cymbidium mosaic virus (CymMV; AAQ03205.1), and PVX (AAV27212.1). These potexviral CPs were then subjected to multiple alignments using the CLUSTAL 2.1 algorithm to identify conserved as well as variable amino acids.

Mutagenesis of PepMV CP. Site-directed mutagenesis of PepMV CP ORF was carried out with an overlapping PCR procedure, using Phusion High-Fidelity DNA polymerase (Thermo Scientific, Lafayette, CO) and appropriate primers (Table 1). To facilitate the incorporation of mutated PepMV CP cDNAs into the pPEP8 infectious clone, three unique restriction enzyme sites (AflII, ClaI, and XhoI) were introduced near the ends of the CP coding sequence, resulting in an intermediary construct designated ACX (Fig. 1B). ACX caused similar diseases in N. benthamiana and tomato plants as the WT PepMV-NE. We then designed mutations based on the multiple alignment results, changing or deleting a selected set of nonconserved amino acid residues at several positions of PepMV CP (Fig. 1; see Results section for details). The plasmids containing mutated PepMV cDNA were then propagated and purified using standard protocols (25). All mutants were sequenced to confirm their identities.

Preparation of in vitro transcripts, and plant inoculation. Plasmids containing full-length cDNAs of WT as well as mutant PepMV were used as templates for in vitro transcription to produce infectious transcripts. Briefly, each of the plasmids was linearized with EagI that cuts the plasmids at the $3^{\prime}$ end of the PepMV cDNA. The linearized plasmid $(1 \mu \mathrm{g})$ was added in a $20-\mu \mathrm{l}$ in vitro transcription reaction containing T7 RNA poly- merase, NTP mix with the $\mathrm{m}^{7} \mathrm{G}$ cap analog, and the appropriate reaction buffer provided by the AmpliCap T7 High Yield MessageMaker Kit (CELLSCRIPT, Inc., Madison, WI). The yield and integrity of the transcripts was confirmed with agarose gel electrophoresis. The transcripts were then used at $50 \mathrm{ng} / \mu \mathrm{l}$ to inoculate $N$. benthamiana leaves. The inoculated plants were reared in a growth chamber under $22^{\circ} \mathrm{C}$, with $14 \mathrm{~h}$ of daylight. Tomato plants grown under similar conditions were inoculated at the cotyledon stage (10 days old) using leaf homogenates of infected $N$. benthamiana plants.

Double-antibody sandwich enzyme-linked immunosorbent assay. Double-antibody sandwich enzyme-linked immunosorbent assay (DAS-ELISA) was used to determine the presence and relative accumulation levels of PepMV and its mutants in systemically infected plants. For each PepMV variant (WT as well as mutants), four infected plants were chosen, and the youngest systemic leaves were collected, processed separately, and assessed for the presence of PepMV using a commercial ELISA kit (Agdia Inc., Elkhart, IN) at 2 and 4 weeks postinoculation (wpi). The results were recorded by measuring the absorbance at $405 \mathrm{~nm}$ with an ELISA plate reader (DTX 880, Multimode Detector; Beckman Coulter Inc., CA), and converted to relative values as described in a previous study (35).

Semiquantitative RT-PCR. Semiquantitative (sq)RT-PCR was carried out using RNA samples extracted from systemically infected leaf tissue to evaluate the relative abundance of PepMV RNA in these samples. Total RNA was extracted at 2 and 4 wpi using the TRIsure reagent (Bioline USA Inc., Taunton, MA), following the manufacturer's instructions. The quality of RNA was confirmed using NanoDrop and agarose gel electrophoresis. The RNA samples were then subjected to DNase treatment to remove residual plant genomic DNA using the Turbo DNA-free kit (Ambion, TX). cDNA synthesis was carried out using $1 \mu \mathrm{g}$ of DNase-treated RNA, $1 \mu \mathrm{M}$ oligo (dT) as the primer, and RevertAid reverse transcriptase (Thermo Scientific, Lafayette, CO) in a $20-\mu l$ volume. Subsequently, $1 \mu \mathrm{l}$ of cDNA was used in PCR reactions that amplified a PepMV-specific, 1,445-bp fragment covering nucleotides 4,936 to 6,380 (sequences of primers

TABLE 1. Oligos used in this study

\begin{tabular}{|c|c|c|}
\hline Oligo name & Sequence & Usage $^{\mathrm{a}}$ \\
\hline \multirow[t]{2}{*}{ T7-PepMV-1F (KpnI) } & GAATTGACGCGTGGTACCTAATACGACTCACTATAGG & \\
\hline & AAAACAAAATAAATAAATAAATATACAAAG & Infectious clone assembly \\
\hline PepMV-6442R (EagI) & CGGTGGCGGCCGCTTTTTTTTTTTTTTTTTTTTTTTTTTT & \\
\hline PepMV-3313F & GAGATATCTTGTTTTTCAATTACCACAA & Infectious clone assembly \\
\hline PepMV-3599R & CATCACTGTTTGTTGCATGAAAGCTGCAA & Infectious clone assembly \\
\hline PepMV-882R & GCGTCTCAAAGTTTCCTCTTTGGAAGA & Sequencing \\
\hline PepMV-1591R & GGTATATGACTGCTAGAAGATGAACA & Sequencing \\
\hline PepMV-4299R & GTGTTAAGTAATTGTGCAGCTCATCA & Sequencing \\
\hline PepMV-6380R & GTAGAAAACCCCACTCTGATTAAGTTTCGAG & Sequencing, RT-PCR \\
\hline PepMV-4936F & GAAGTCTTGGACTTTGTTTCTGACCA & Sequencing, RT-PCR \\
\hline PepMV-5091R & GGTGTGTCTAGTCAGAGAGATGAA & Sequencing \\
\hline PepMV-5620F (ClaI, delPDT) & TCAATAATCGATCATGACACCTGTTGCTGCCACTTCA & Mutagenesis \\
\hline PepMV-6361R (XhoI, EL>PP) & TTAAGTCTCGAGTGTTTATGGAGGAGGGGGTGCGTCTA & Mutagenesis \\
\hline PepMV-5814F (TA>KD) & TAGGCAAAATCTTTAAAGATATGGGCCTTGCCGCCAA & Mutagenesis \\
\hline PepMV-5567AflII-F & CCTTGGAACGGCTTAAGTTTTCCTAAATTTGA & Mutagenesis \\
\hline PepMV-5567AflII-R & TCAAATTTAGGAAAACTTAAGCCGTTCCAAGG & Mutagenesis \\
\hline NbACT-F2 & CAGCCACACTGTCCCAATTTATGAG & RT-PCR of Nicotiana benthamiana actin \\
\hline $\mathrm{Nt} / \mathrm{NbACT}-\mathrm{R}$ & CACCTTAATTTTCATACTGCTTGGA & RT-PCR of $N$. benthamiana actin \\
\hline Tomato actin-F & GTTGGTGACGAGGCTCAATCCAAA & RT-PCR of tomato actin \\
\hline Tomato actin-R & CTGTTCATAGTCAAGAGCAATGTAAG & RT-PCR of tomato actin \\
\hline
\end{tabular}

${ }^{\mathrm{a}} \mathrm{RT}-\mathrm{PCR}=$ reverse-transcription polymerase chain reaction. 
compiled in Table 1). The PCR enzyme used was the EconoTaq Plus Green 2X Master Mix (Lucigen, Middleton, WI). Additionally, actin-specific fragments of $N$. benthamiana or tomato were amplified in parallel to serve as controls to ensure similar amounts of RNA were used for different samples (primers listed in Table 1). Unless stated otherwise, the conditions for PCR were one denaturing step at $94^{\circ} \mathrm{C}$ for $4 \mathrm{~min} ; 30$ cycles of amplification at $94^{\circ} \mathrm{C}$ for $30 \mathrm{~s}, 56^{\circ} \mathrm{C}$ for $30 \mathrm{~s}$, and $72^{\circ} \mathrm{C}$ for $60 \mathrm{~s}$; and a final extension step at $72^{\circ} \mathrm{C}$ for $7 \mathrm{~min}$. The resulting PCR products were examined with agarose gel electrophoresis.

Cross-protection experiments. For all constructs, inocula were propagated by inoculating the third or fourth true leaves of young $N$. benthamiana plants with in vitro transcripts of the corresponding constructs (36). The inoculated leaves (ILs) were harvested as soon as systemic symptoms became visible $(\approx 4$ days postinoculation [dpi]) and stored at $-80^{\circ} \mathrm{C}$ for future use. For testing cross-protection, $N$. benthamiana or tomato plants were inoculated with the primary inocula prepared from the stored ILs at the three-leaf and cotyledon stage, respectively. The secondary inocula were then applied onto upper young leaves of these plants at 2 weeks after primary inoculations.

\section{RESULTS}

Characterization of a new PepMV isolate from Nebraska. A symptomatic tomato plant received by the Morris lab at the University of Nebraska-Lincoln in 2006 was found to contain a mechanically transmissible entity infectious on $N$. benthamiana plants. Electron microscopy revealed virus particles of flexuous rod shape, $\approx 600 \mathrm{~nm}$ in length (Fig. 1A), suggestive of potexviruses. Purification of viral dsRNA, followed by cDNA synthesis with random hexamers, yielded two cDNA fragments of $\approx 500 \mathrm{bp}$ with sequences highly similar to known PepMV isolates (data not shown). The full-length sequence of PepMV-NE was then obtained by sequencing overlapping PCR fragments (sequences of primers compiled in Table 1) and deposited to GenBank under the accession number KF281605. Excluding the polyA tail, the genome of PepMV-NE is 6,410 nucleotides (nt). Interestingly, its sequence is most closely related, albeit not identical, to isolates from Hungary, Spain, The Netherlands, and other European countries (99\% at the nucleotide level). Nevertheless, it differs from these PepMV isolates at a minimum of $44 \mathrm{nt}$ positions. At the amino acid sequence level, PepMV-NE differs from the closest known PepMV isolate (PepMV-H from Hungary; AM491606.1) in the 1,439-amino-acid (aa) RdRp by 14 aa. Elsewhere in the PepMV genome, the predicted TGB1, 2, 3, and CP peptides differ from those of PepMV-H at one, three, one, and two amino acid positions, respectively. These results indicate that PepMV-NE is a new PepMV isolate very closely related to PepMV isolates of European origins.

Based on their origins and sequence similarities, previously reported PepMV isolates were grouped into five genotypes: LP, EU, US1, US2, and CH2 (12). To determine the genotype of PepMV-NE, we compared the full-length nucleotide sequence of PepMV-NE with representative members of the five genotypes. PepMV-NE is 96, 99, 82, 80, and 79\% identical to AJ606361.1 (genotype LP), AJ438767 (EU), AY509926 (US1), AY509927 (US2), and DQ000985 (CH2), respectively. Hence, it should be considered as an isolate of the genotype EU.

An infectious clone of PepMV-NE was generated by cloning its full-length cDNA, together with a 30-nt polyA tail at the $3^{\prime}$ end, into a modified pBluescript SK plasmid, downstream of the T7 promoter, giving rise to pPEP8. Capped in vitro transcripts derived from pPEP8 were highly infectious in $N$. benthamiana as well as tomato, causing clearly visible symptoms such as mosaic, misshaped, and bulged leaves in $N$. benthamiana and some cultivars of tomato (e.g., 'Rutgers' and 'Yellow Pear'). Thus, we have succeeded in obtaining an infectious clone of PepMV-NE.
This clone was subsequently used to engineer attenuated crossprotective PepMV variants.

Comparison of PepMV CP to other potexviral CPs identifies variable amino acid residues as candidates for mutagenesis. We chose PepMV $\mathrm{CP}$ as the target for mutagenesis because $\mathrm{CP}$ is not needed for viral genome replication yet is required for virion assembly as well as intraplant spread. As a result, changes in $\mathrm{CP}$ could potentially modulate disease symptoms without jeopardizing viral multiplication. In addition, its ORF does not overlap with other viral genes, permitting straightforward manipulations. To ensure that our modification strategy is applicable to most PepMV isolates of different genotypes, we first compared the CPs of PepMV-NE and five other PepMV isolates belonging to LP, EU, US1, US2, and $\mathrm{CH} 2$ genotypes (12). The CPs of these six PepMV isolates are highly similar, except for the $\mathrm{N}$ terminus (Fig. 1C). Indeed, PepMV-NE CP is $100 \%$ identical to the LP and EU isolates, and differs from the US1, US2, and $\mathrm{CH} 2$ isolates at only 7, 18, and 18 aa positions out of a total of 237 aa. Furthermore, all but four of these variations occur within the Nterminal 26 aa, which are thought to be partially exposed on the surface of the virions (18). The high level of sequence conservation among CPs of diverse PepMV isolates further justifies CP as an ideal target for engineered attenuation.

Next, to identify amino acid residues in PepMV-NE CP that could tolerate alterations, its amino acid sequence was compared with its counterparts in five other closely related potexviruses: NarMV, AlsVX, LetVX, CymMV, and PVX. Potexviral CPs are also well conserved, except for a short N-terminal region (Fig. 1D). Nevertheless, additional less-conserved short amino acid spans could be identified elsewhere upon closer inspection. Based on the alignment results, four of the less-conserved spans, three of them conserved among all PepMV isolates (Fig. 1C and D, boxed amino acids), were chosen for mutagenesis. Note that, in order to accommodate changes within PepMV CP, an intermediary construct designated ACX was created by flanking the CP coding region with newly introduced $A f l I I, C l a I$, and XhoI sites (Fig. 1B). Systemic symptoms caused by ACX were indistinguishable from WT PepMV in both $N$. benthamiana and tomato plants.

We then adopted the straightforward strategy of replacing the boxed amino acids (Fig. 1C and D) with their counterparts in PVX, resulting in mutants KD, VC, and PP (Fig. 1B). In addition, a $\triangle$ PDT mutant was created by deleting three nonconserved amino acids at the $\mathrm{N}$ terminus of PepMV (Fig. 1B and C). Finally, combining the changes in $\triangle \mathrm{PDT}$ and $\mathrm{PP}$, as well as those in $\mathrm{KD}$ and $\mathrm{VC}$, gave rise to the $\triangle \mathrm{PDT} / \mathrm{PP}$ and $\mathrm{KD} / \mathrm{VC}$ mutants, respectively (Fig. 1B). Altogether, six PepMV mutants were generated and assessed for their replication, systemic spread, symptom attenuation, as well as cross-protection potentials in host plants.

PepMV CP mutants show delayed or attenuated infections in $N$. benthamiana. To evaluate the infectivity of the six PepMV mutants, in vitro transcripts of these mutants were used to infect $N$. benthamiana plants, with WT pPEP8 transcripts as the positive control. All mutants except for KD and KD/VC developed clearly visible symptoms exemplified by mosaic, bulged and misshapen systemic leaves, and stunted stature at 4 wpi (Fig. 2A). However, the time at which symptoms became visible differed from mutant to mutant. Although plants infected with WT PepMV, ACX, or $\triangle \mathrm{PDT}$ showed the first signs of systemic infection as early as $4 \mathrm{dpi}$, those infected with VC, PP, and $\triangle \mathrm{PDT} / \mathrm{PP}$ mutants remained asymptomatic until 2 wpi, at which point severe symptoms progressed relatively quickly, leading to stunting of infected plants by 4 wpi. Finally, plants inoculated with KD/VC never developed discernible symptoms.

Interestingly, the KD-infected $N$. benthamiana plants showed signs of systemic infection relatively early (4 dpi) but those symptoms were considerably milder than those caused by most other mutants (except for KD/VC). Indeed, these symptoms continued to moderate so that, by $4 \mathrm{wpi}$, the $\mathrm{KD}$-infected plants 
were almost indistinguishable from mock controls (Fig. 2A). These results strongly suggest that the KD mutant was able to replicate in $N$. benthamiana cells and spread systemically yet caused milder disease symptoms, making this mutant a strong candidate for inducing cross-protection.

Mutant KD accumulates low levels of viral RNA in systemic leaves of infected plants. The levels of mutant viral RNAs in infected tissues were assessed using sqRT-PCR at two time points: 2 and 4 wpi. In these experiments, a 510-bp fragment derived from an $N$. benthamiana actin ( $\mathrm{Nb}$ actin) mRNA was used as a control to ensure that a similar amount of total RNA was used in all samples (Fig. 2B). At 2 wpi, viral RNA was barely detectable in plants infected with $\mathrm{VC}$ and undetectable in those infected with $\triangle \mathrm{PDT} / \mathrm{PP}$ or $\mathrm{KD} / \mathrm{VC}$ (Fig. $2 \mathrm{~B}$, top two panels, lanes 6,8 , and 9). However, it was easily detectable in plants infected with the $\triangle \mathrm{PDT}, \mathrm{KD}$, and PP single mutants (Fig. 2B, top two panels, lanes 4, 5, and 7). Notably, the level of KD viral RNA was

\section{A}
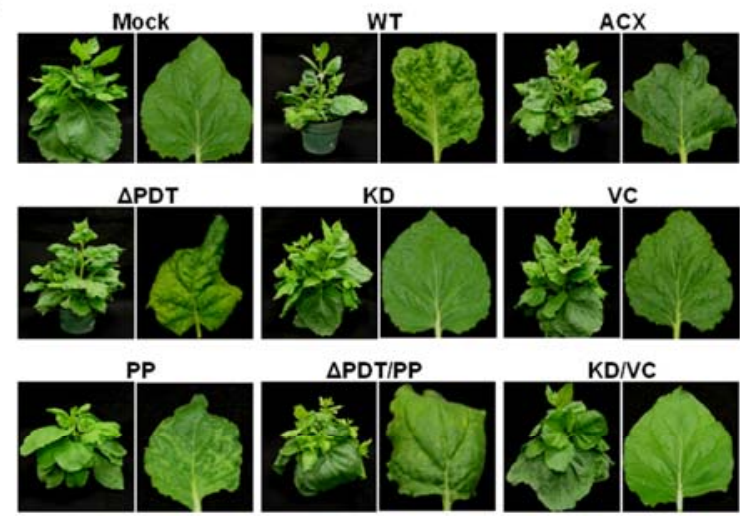

$\triangle P D T / P P$
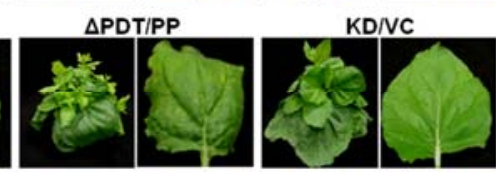

B

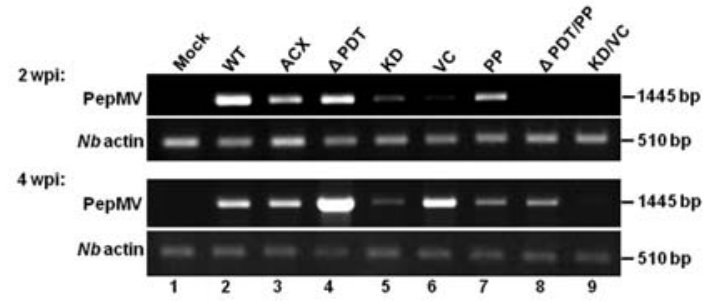

C

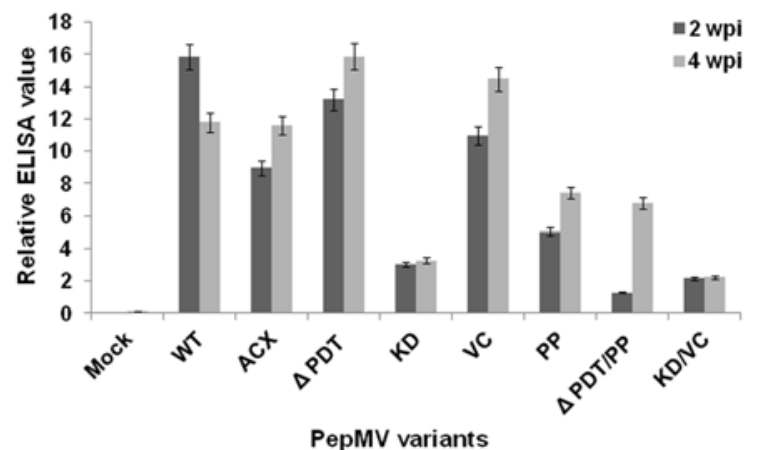

Fig. 2. Infectivity of six Pepino mosaic virus (PepMV) mutants with altered viral capsid proteins (CPs). A, Systemic symptoms induced by the mutants in Nicotiana benthamiana. For each PepMV variant, a pair of images were shown, with the left one representing an infected plant and the right a close-up of a systemic leaf. B, Detection of viral RNA in systemic $N$. benthamiana leaves using semiquantitative reverse-transcription polymerase chain reaction at 2 (top panel) and 4 (third panel) weeks postinoculation (wpi). The two $N$. benthamiana $(\mathrm{Nb})$ actin panels serve as controls for RNA amounts in different samples. C, Detection of PepMV CP in systemic $N$. benthamiana leaves using double-antibody sandwich enzyme-linked immunosorbent assay at 2 (dark gray) and 4 (light gray) wpi. Relative readings were used to produce the graph. Error bars represent variations among four samples. lower than that of $\triangle \mathrm{PDT}$ and PP (Fig. 2B, top panel, compare lane 5 with lanes 4 and 7).

By 4 wpi, levels of KD/VC-specific viral RNA remained extremely low (Fig. 2B, third panel, lane 9; data not shown). By this point in time, however, RNA from all other mutants could be detected in infected tissues. Interestingly, the $\triangle \mathrm{PDT}$ and VC mutants appeared to be more rigorous than even WT PepMV (Fig. 2B, third panel; compare lanes 4 and 6 with lane 2). In contrast, the level of KD-specific viral RNA remained low compared with other mutants, except for KD/VC. The consistently low level of KD viral RNA in systemically infected leaves was in agreement with the mild diseases caused by this mutant, further suggesting KD as an attenuated PepMV variant with cross protection potentials.

Mutant KD produces substantially lower levels of $\mathrm{CP}$ than WT PepMV. The sqRT-PCR approach provided a direct measurement of viral RNA levels. However, due to the relatively high costs of the multistep procedure, only one plant per treatment group could be assessed. As a result, potential plant-toplant variations could be missed. To address these shortcomings, we then used DAS-ELISA to evaluate the levels of PepMV CP in multiple infected plants. For every PepMV mutant, four infected plants were chosen for DAS-ELISA experiments. Additionally, the experiments were repeated three times with three different sets of plants, yielding similar results. One typical set of results was shown in Figure 2C. To permit comparisons between different sets of experiments, the optical readings of ELISA results were converted to relative values by dividing the readings of test wells with the average of readings of negative control wells, with a relative value of two or above counted as positive (35). Although the CP of most mutants accumulated to levels that were at least fivefold higher than negative controls, that of both $\mathrm{KD}$ and $\mathrm{KD} / \mathrm{VC}$ mutants was consistently low, at approximately threeand twofold higher than the negative controls, respectively (Fig. 2C). These results further illustrated the reduced infectivity of the KD mutant.

Mutant KD provides effective protection against subsequent infection by WT PepMV in $N$. benthamiana. We next evaluated the potential of mutant KD to cross protect host plants against the secondary infection by WT PepMV. To this end, six $N$. benthamiana plants were first inoculated with mutant $\mathrm{KD}$ and reared for 2 weeks to allow for systemic spread. The plants were then challenged with WT PepMV on upper young leaves and observed on a daily basis for symptom development. This set of experiments was repeated three times with similar results. Although WT PepMV alone, introduced as either the primary or superinoculum, caused severe stunting of the infected plants, KD as the primary inoculum was correlated with plant sizes almost indistinguishable from mock-inoculated plants (Fig. 3A). Most strikingly, superinfection of KD-infected plants with WT PepMV did not measurably change the appearance of the plants (Fig. 3A, right-most plant).

The above observations were further corroborated with DASELISA. Although PepMV CP titers in plants infected with WT PepMV alone (as primary or secondary inoculum) were consistently high (approximately fivefold higher than in plants infected with KD alone), they remained low in KD/WT superinfected plants for at least 4 weeks after superinfection (Fig. 3B). These results strongly suggest that the presence of KD mutant genome in $N$. benthamiana inhibited the replication of the WT PepMV genome, thus repressing the severe symptoms associated with the presence of WT PepMV.

To evaluate whether WT PepMV gRNA was present in KD/WT superinfected plants, a DraI site introduced into the KD genome at the time of the mutant creation was utilized to distinguish WT PepMV and mutant KD. An RT-PCR fragment spanning nucleotides 4,936 to 6,442 of WT PepMV genome (1,506 bp) contains one DraI site at nucleotide 6,344 and, thus, is expected to give rise to two fragments (1,408 and $98 \mathrm{bp}$ ) (Fig. 3C, top diagram) 
upon DraI digestion. Although the 98-bp fragment was nearly invisible due to its small size, its presence can be inferred from the slight difference of the WT-derived fragment before and after DraI digestion (1,506 versus 1,408 bp) (Fig. 3C, compare lane 2 with lanes 3 and 4). Conversely, the same PCR fragment derived from KD genome is expected to produce three DraI fragments (892, 517, and $98 \mathrm{bp}$ ) due to the additional DraI site in KD mutant (nucleotide 5,827) (Fig. 3C, second diagram). Accordingly, detection of the 1,408-bp DraI fragment in KD/WT superinfections would indicate the presence of WT PepMV genome. The PCR fragment derived from KD/WT superinfected plants was completely digested into three fragments (Fig. 3C, lane 6), thus giving rise to the same band pattern as the KD-specific fragment (lane 5). These results indicated that WT PepMV was unable to accumulate to detectable levels in plants preinoculated with KD. To conclude, mutant KD readily cross-protected $N$. benthamiana plants against superinfection of WT PepMV.

Mutant KD retains its cross-protection capacity in tomato plants. We then assessed the cross-protection capacity of mutant KD in tomato, the major crop host of PepMV. For this purpose, we first screened 14 tomato cultivars ('Horizon', Rutgers, 'FL7600', 'FL47R', 'SUN1642', 'NC84173', 'OH981067', 'PI128216', 'LA2204', 'LA2167', 'LA407', Yellow Pear, 'LA2204', and 'Livingston Gold Ball') for the ones highly susceptible to PepMV-NE by inoculating six seedlings of each cultivar with this virus. Yellow Pear was chosen for subsequent experiments because it developed the distinctive upward curling of leaf margins and stunting of the adult plant upon PepMV inoculation (Fig. 4A, second and third panels). The superinfection was then carried out on this variety of tomato plants in the same manner as on $N$. benthamiana plants. WT PepMV-infected plants (again as both primary inoculums or superinoculum on mock plants) developed the characteristic symptoms of upward curled leaf margins, accompanied by stunting, whereas those infected with KD remained symptomless even after being superinfected with WT PepMV (Fig. 4A). Accordingly, DAS-ELISA experiments confirmed low PepMV CP titers in KD as well as KD/WT superinfected plants (Fig. 4B). Again, DraI digestion of RT-PCR products confirmed the absence of WT PepMV genome in the superinfected tomato plants (Fig. 4C).

Mutant KD is stable for at least five passages in $N$. benthamiana. In order to ensure that mutant KD does not easily revert to WT PepMV, we tested its stability by subjecting this mutant to multiple rounds of infections in $N$. benthamiana plants. Mutant $\mathrm{KD}$ was passaged in $N$. benthamiana for five generations by using the leaf extracts of infected plants to inoculate five new plants every 30 days. Mild symptoms were consistently observed on all inoculated plants throughout the 5-month passaging period (data not shown). Additionally, the systemic leaf tissues were collected from plants of every passage and subjected to RT-PCR followed by DraI digestion to confirm the identity of the mutation. The original KD mutant was the predominant viral variant in all three plants of the first, third, and fifth passages analyzed (Fig. 5), thus confirming the stability of the mutant at the genome level. These results were also confirmed by direct sequencing of the RT-PCR products (data not shown).

\section{DISCUSSION}

PepMV is a serious viral pathogen of glasshouse tomato production, for which no genetic resistance resource is identified in tomato germplasm collections. Although some accessions of other Solanum spp. (e.g., Solanum habrochaites and S. pseudocapsicum L.) were found to be resistant to PepMV, cross-species breeding of these resistance genes into tomato could be difficult and time consuming $(17,29)$. Consequently, cross-protection has been used in commercial productions to manage PepMV-caused tomato diseases (27). However, thus far, this practice has gen- erated mixed results, due primarily to the unpredictability of the field-isolated cross-protective PepMV variants. In the current study, we report the successful generation of a cross-protective PepMV mutant through knowledge-based attenuation of a severe PepMV isolate (PepMV-NE). This mutant, referred to as mutant $\mathrm{KD}$, was one of the six PepMV mutants produced by introducing site-directed mutations within the PepMV CP gene. Consideration

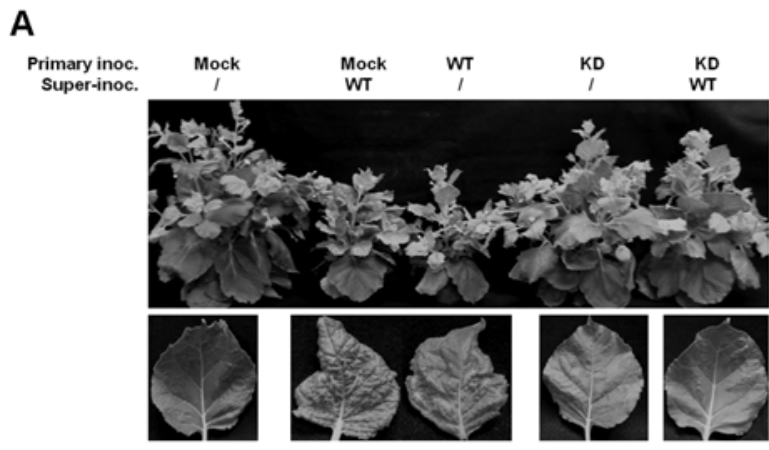

B

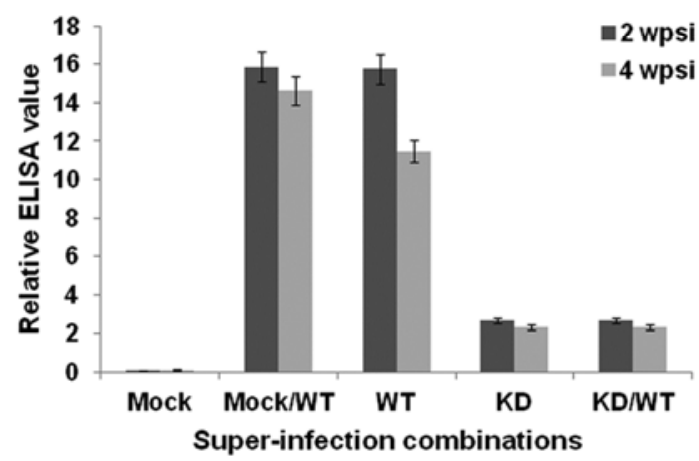

C
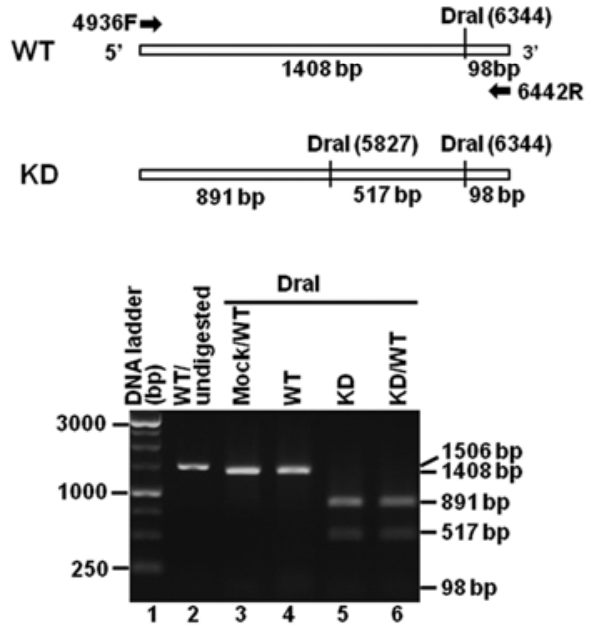

Fig. 3. Pepino mosaic virus (PepMV) mutant KD cross-protects Nicotiana benthamiana from superinfection by wild-type (WT) PepMV. A, Representative plants (top) and leaves (bottom) of superinfected $N$. benthamiana. Plants were first infected with buffer only (Mock), WT PepMV, or mutant KD (primary inocula), followed by superinoculation with WT PepMV 2 weeks later. The images were taken at 4 weeks post superinfection (wpsi). B, Detection of PepMV capsid protein (CP) in systemic leaves of superinfected plants at 2 and 4 wpsi using double-antibody sandwich enzyme-linked immunosorbent assay. C, Determination of the identity of PepMV variants in superinfected leaves. The two diagrams on the top depict the reversetranscription polymerase chain reaction-amplified sections of WT and KD cDNA (nucleotides 4,936 to 6,432), and their DraI-digested fragments and corresponding sizes. Lane 6 shows that the WT-specific cDNA was undetectable in superinfected plants. 
of the $\mathrm{CP}$ gene as a target for virus attenuation was based on previous reports suggesting that CPs of plant RNA viruses play important roles in the infection process (3). In addition to protecting viral genomes by forming protective capsids, they can affect viral pathogenesis through its implication in almost all stages of the viral life cycle, beginning with processes that enhance virus entry into plant cells to those that facilitate the transmission of viruses from plant to plant. For example, PepMV $\mathrm{CP}$ has been shown to interact with a tomato heat shock protein and thereby influence viral symptoms (19).

In our study, the specific candidate sites within PepMV CP were first identified by comparing the PepMV CP sequence with that of five closely related potexviruses. This allowed us to locate a number of variable amino acid pairs or clusters within the relatively conserved potexviral CPs. We reason that these amino acid variations could correlate with the adaptation of a particular potexvirus to its corresponding natural hosts. Conversely, replacing them with their counterparts in a different potexvirus could lead to reduced fitness of that virus in its natural hosts without abolishing its infectivity.

Compared with WT PepMV, mutant KD contains two amino acid changes within PepMV CP (Thr to Lys at amino acid 66 and Ala to Asp at amino acid 67, respectively). It induced very mild disease symptoms on both $N$. benthamiana and the crop host tomato, accompanied by lower accumulation of viral RNA and CP. More importantly, mutant KD protected both $N$. benthamiana and tomato plants against secondary infection with WT PepMV and, therefore, constitutes an ideal inducer of cross-protection. Thus, we have succeeded in developing a relatively simple procedure for engineering attenuated plant viruses capable of cross protection. One obvious advantage of this procedure is its relative speed, allowing for swift response to future emerging viral diseases. Generation of cross-protective viral variants through site-directed mutagenesis has been reported for ZYMV by two independent groups $(8,15)$. However, both of those studies used the sequences of naturally occurring, mild viral variants to

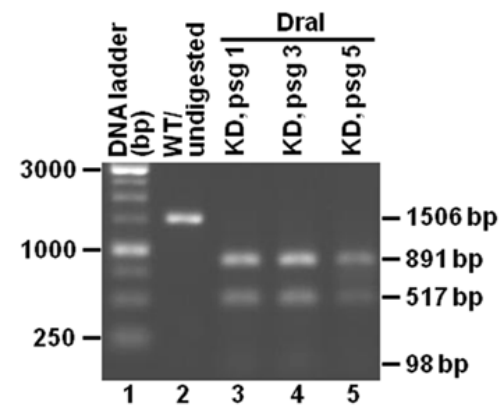

Fig. 5. Mutant KD is stable for at least five passages (psgs) in Nicotiana benthamiana. The stability of KD was first assessed with DraI digestion of a reverse-transcription polymerase chain reaction (PCR) fragment of Pepino mosaic virus (PepMV) spanning the KD mutation (please see the top two diagrams in Figure $3 \mathrm{C}$ for the expected sizes of the digested fragments), which yielded KD-specific fragments. The PCR fragments were also sequenced to confirm the digestion results (data not shown). Lanes 3, 4, and 5 show digestion results obtained from representative plants of the first, third, and fifth passages, respectively.

\section{A Primary Inoc. Super-inoc.}
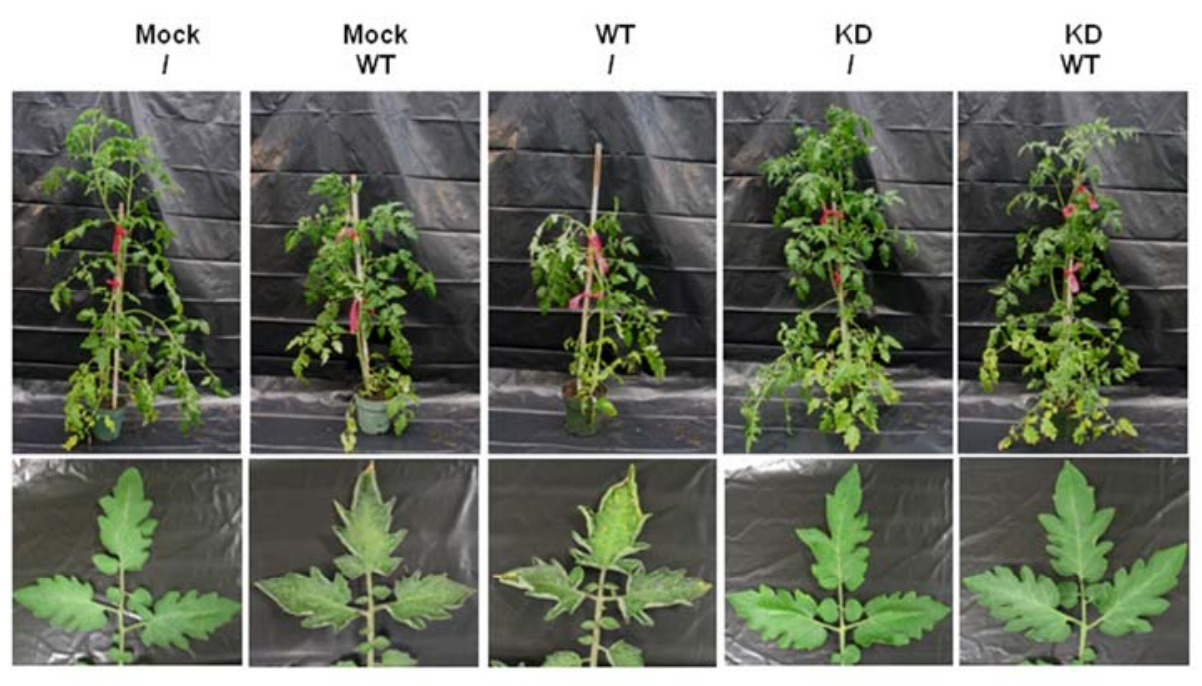

B

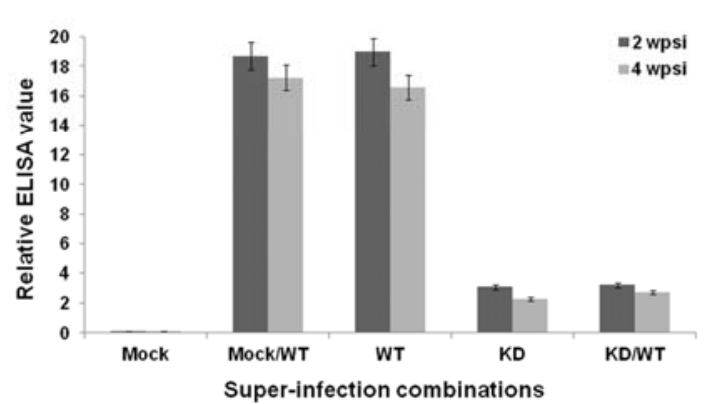

C

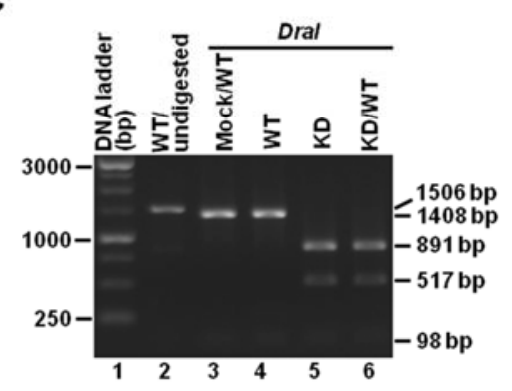

Fig. 4. Mutant KD cross-protects tomato from superinfection by wild-type (WT) Pepino mosaic virus (PepMV). A, Representative plants (top) and leaves (bottom) of superinfected tomato ('Yellow Pear'). Plants were first infected with buffer only (Mock), WT PepMV, or mutant KD (primary inocula), followed by superinoculation with WT PepMV 2 weeks later. The images were taken at 4 weeks post superinfection (wpsi). B, Detection of PepMV capsid protein (CP) in systemic leaves of superinfected tomato plants at 2 and 4 wpsi using double-antibody sandwich enzyme-linked immunosorbent assay (ELISA). C, Determination of the identity of PepMV variants in superinfected leaves. Please see the top two diagrams in Figure 3C for the expected sizes of the digested fragments. Lane 6 shows that the WT-specific cDNA was undetectable in superinfected plants. 
guide their mutagenesis. By contrast, our approach is completely independent of any knowledge of known mild isolates and, hence, has the potential to be widely applicable to other viruses.

Research on the mechanisms of cross-protection suggested that a number of defense pathways could contribute to the protection. One possibility is homology-based RNA silencing, which could generate small interfering RNAs (siRNAs) from the crossprotective variant, and use these siRNAs to thwart the invasion of more severe viral variants $(22,23)$. A second possibility is $\mathrm{CP}$ mediated protection $(2,28)$. Alternatively, competition for certain unknown factors of the plant host could also contribute (38). Finally, recently published results suggest that cross protection might be caused by certain virus-encoded functions $(6,7)$. Although it remains to be determined which mechanism is at play for mutant KD-mediated cross-protection against PepMV, we showed that the superinfected WT PepMV was undetectable in plants preinoculated with $\mathrm{KD}$, thus suggesting a near complete exclusion of the former. It is possible that replication of the crossprotective viral variant exhausts important host resources that are required for virus replication or completely occupies sites required by the challenge virus. The preexisting $\mathrm{CP}$ from mutant $\mathrm{KD}$ could also inhibit uncoating and replication of the WT virus, as has previously been shown to occur with TMV $(2,26)$. In addition, the attenuated KD mutant might induce the expression of specific defense factors that resist infection by WT PepMV through a mechanism yet to be fully elucidated.

Cross-protection as a form of viral resistance offers great promise in plant disease management. If properly utilized, it could be effective in combating many plant viruses in many different crop species. However, the unavailability of naturally occurring mild strains for many viruses remains a great obstacle for its adoption. Therefore, in order for cross-protection to succeed, it is important to develop rapid and reliable procedures for generating attenuated viruses capable of cross protection. By demonstrating the successful engineering of an attenuated PepMV variant with cross protection potentials, we succeeded in developing a practical, knowledge-based approach for the production of cross-protective plant virus variants. The data presented here provide a foundation for further investigations and field trials to demonstrate the feasibility of using mutant KD for widespread control of PepMV. Our approach to engineering attenuated viruses is novel, rapid, and cost effective and, hence, is likely to be applicable to other plant viruses, leading to improved productivity in major crops prone to virus diseases.

\section{ACKNOWLEDGMENTS}

We thank M. Ellis for his insightful advice, all members of the Qu lab for valuable suggestions, M. G. Redinbaugh and L. Stewart and other members of the United States Department of Agriculture-Agricultural Research Service Corn and Soybean Research Unit for sharing equipment, and K.-S. Ling for providing us with the seed of Horizon and Rutgers tomato. This research was supported, in part, by a Fulbright grant to G. M. Chewachong.

\section{LITERATURE CITED}

1. Adams, M. J., Antoniw, J. F., Bar-Joseph, M., Brunt, A. A., Candresse, T., Foster, G. D., Martelli, G. P., Milne, R. G., Zavriev, S. K., and Fauquet, C. M. 2004. The new plant virus family Flexiviridae and assessment of molecular criteria for species demarcation. Arch. Virol. 149:1045-1060.

2. Bendahmane, A., Chen, I., Asurmendi, S., Bazzini, A. A., Szecsi, J., and Beachy R. N. 2007. Coat protein-mediated resistance to TMV infection of Nicotiana tabacum involves multiple modes of interference by coat protein. Virology 366:107-116.

3. Bol, J. F. 2008. Role of capsid proteins. Methods Mol. Biol. 451:21-31.

4. Cho, J. J., Ullman, D. E., Wheatley, E., Holly, J., and Gonsalves, D. 1992. Commercialization of ZYMV cross protection for zucchini production in Hawaii. (Abstr.) Phytopathology 82:1073.

5. Costa, A. S., and Muller, G. W. 1980. Tristeza control by cross protection. Plant Dis. 64:538-541.
6. Folimonova, S. Y. 2012. Superinfection exclusion is an active viruscontrolled function that requires a specific viral protein. J. Virol. 86:55545561.

7. Folimonova, S. Y., Robertson, C. J., Shilts, T., Folimonova, A. S., Hilf, M. E., Garnsey, S. M., and Dawson, W. O. 2010. Infection with strains of Citrus tristeza virus does not exclude superinfection by other strains of the virus. J. Virol. 84:1314-1325.

8. Gal-On, A. 2000. A point mutation in the FRNK motif of the potyvirus helper component-protease gene alters symptom expression in cucurbits and elicits protection against the severe homologous virus. Phytopathology 90:467-473.

9. Gal-On, A., Katsir, P., and Yongzang, W. 2000. Genetic engineering of attenuated viral cDNA of Zucchini yellow mosaic virus for protection of cucurbits. Acta Hortic. 510:343-347.

10. Hanssen, I. M., Gutiérrez-Aguirre, I., Paeleman, A., Goen, K., Wittemans, L., Lievens, B., Vanachter, A. C. R. C., Ravnikar, M., and Thomma, B. P. H. J. 2010. Cross-protection or enhanced symptom display in greenhouse tomato co-infected with different Pepino mosaic virus isolates. Plant Pathol. 591:13-21.

11. Hanssen, I. M., Paeleman, A., Wittemans, L., Goen, K., Lievens, B., Bragard, C., Vanachter, A. C. R. C., and Thomma, B. P. H. J. 2008. Genetic characterization of Pepino mosaic virus isolates from Belgian greenhouse tomatoes reveals genetic recombination. Eur. J. Plant Pathol. 121:131-146.

12. Hanssen, I. M., and Thomma, B. P. H. J. 2010. Pepino mosaic virus: A successful pathogen that rapidly evolved from emerging to endemic in tomato crops. Mol. Plant Pathol. 11:179-189.

13. Jones, R., Koenig, R., and Lesemann D. 1980. Pepino mosaic virus, a new potexvirus from pepino (Solanum muricatum). Ann. Appl. Biol. 94:61-68.

14. Lecoq H. 1998. Control of plant virus diseases by cross protection. Pages 33-40 in: Plant Virus Disease Control. A. Hadidi, R. K. Khaterpal, and H. Koga-Nezawah, eds. American Phytopathological Society Press, St. Paul, MN.

15. Lin, S. S., Wu, H. W., Jan, F. J., Hou, R. F., and Yeh, S. D. 2007. Modifications of the helper component-protease of Zucchini yellow mosaic virus for generation of attenuated mutants for cross protection against severe infection. Phytopathology 97:287-296.

16. Ling, K.-S. 2008. Pepino mosaic virus on tomato seed: Virus location and mechanical transmission. Plant Dis. 92:1701-1705.

17. Ling, K.-S., and Scott, J. W. 2007. Sources of resistance to Pepino mosaic virus in tomato accessions. Plant Dis. 91:749-753.

18. Lukashina, E., Badun, G., Fedorova, N., Ksenofontov, A., Nemykh, M., Serebryakova, M., Mukhamedzhanova, A., Karpova, O., Rodionova, N., Baratova, L., and Dobrov, E. 2009. Tritium planigraphy study of structural alterations in the coat protein of Potato virus $X$ induced by binding of its triple gene block 1 protein to virions. FEBS J. 276:70067015.

19. Mathioudakis, M. M., Veiga, R., Ghita, M., Tsikou D., Medina, V., Canto, T., Makris, A. M., and Livieratos, I. C. 2012. Pepino mosaic virus capsid protein interacts with a tomato heat shock protein cognate 70 . Virus Res. 163:28-39.

20. Morris, T. J., and Dodds, J. A. 1979. Isolation and analysis of doublestranded RNA from virus-infected plants. Phytopathology 69:854-858.

21. Nishiguchi, M., and Kobayashi, K. 2011. Attenuated plant viruses: Preventing virus diseases and understanding the molecular mechanism. J. Gen. Plant Pathol. 77:221-229.

22. Ratcliff, F., Harrison, B. D., and Baulcombe, D. C. 1997. A similarity between viral defense and gene silencing in plants. Science 276:15581560.

23. Ratcliff, F., MacFarlane S. A., and Baulcombe, D. C. 1999. Gene silencing without DNA: RNA mediated cross protection between viruses. Plant Cell 11:1207-1216.

24. Salaman, R. N. 1933. Protective inoculation against a plant virus. Nature (London) 131:468.

25. Sambrook, J., and Russell, D. W. 2001. Molecular Cloning: A Laboratory Manual. Cold Spring Harbor Laboratory Press, Cold Spring Harbor, NY.

26. Sanders, P. R., Sammons, B., Kaniewski, W., Haley, L., Layton, J., LaVallee, B. J., Delannay, X., and Tumer, N. E. 1992. Field-resistance of transgenic tomatoes expressing the tobacco mosaic virus or tomato mosaic virus coat protein genes. Phytopathology 82:683-690.

27. Schenk, M. F., Hamelink, R., van der Vlugt, R. A. A., Vermunt, A. M. W., Kaarsenmaker, R. C., and Stijger, I. C. C. M. M. 2010. The use of attenuated isolates of Pepino mosaic virus for cross-protection. Eur. J. Plant Pathol. 127:249-261.

28. Sherwood, J. L. 1987. Demonstration of the specific involvement of coat protein in tobacco mosaic virus (TMV) cross protection using a TMV coat protein mutant. J. Phytopathol. 118:358-362.

29. Soler-Aleixandre, S., Lopez, C., Cebolla-Cornejo, J., and Nuez, F. 2007. Sources of resistance to Pepino mosaic virus (PepMV) in tomato. HortScience 42:40-45. 
30. Soler-Aleixandre, S., Lopez, C., Diez, M., de Castro, A., and Nuez, F. 2005. Association of Pepino mosaic virus with tomato collapse. J. Phytopathol. 153:464-469.

31. Verchot-Lubicz, J., Ye, C. M., and Bamunusinghe, D. 2007. Molecular biology of potexviruses: Recent advances. J. Gen. Virol. 88:1643-1655.

32. Walkey, D. G. A., Lecoq, H., Collier, R., and Dobson, S. 1992. Studies on the control of zucchini yellow mosaic virus in courgettes by mild strain protection. Plant Pathol. 41:762-771.

33. Wallace, J. M. 1940. Evidence of passive immunization of tobacco, Nicotiana tabacum, from the virus of curly top. Phytopathology 30:673-679.

34. Yeh, S. D., and Gonsalves, D. 1984. Evaluation of induced mutants of Papaya ringspot virus for control by cross protection. Phytopathology 74:1086-1091.
35. Zhang, X., Sato, S., Ye, X., Dorrance, A. E., Morris, T. J., Clemente, T. E., and Qu, F. 2011. Robust RNAi-based resistance to mixed infection of three viruses in soybean plants expressing separate short hairpins from a single transgene. Phytopathology 101:1264-1269.

36. Zhang, X. C., Zhang, X. F., Singh, J., Li, D., and Qu, F. 2012. Temperature-dependent survival of Turnip crinkle virus-infected Arabidopsis plants relies on an RNA silencing-based defense that requires DCL2, AGO2, and HEN1. J. Virol. 86:6847-6854.

37. Ziebell, H., and Carr, J. P. 2010. Cross-protection: A century of mystery. Adv. Virus Res. 76:211-264.

38. Ziebell, H., Payne, T., Berry, J. O., Walse, J. A., and Carr, J. P. 2007. A Cucumber mosaic virus mutant lacking the $2 \mathrm{~b}$ counter-defence protein gene provides protection against wild type strains. J. Gen. Virol. 88:2862-2871. 